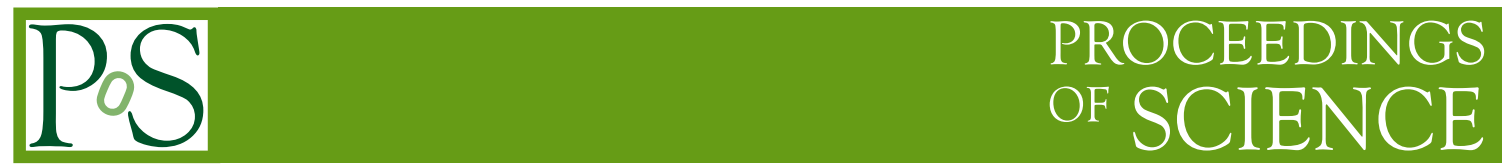

\title{
Prospects of $\mathrm{CP}$ violation in $\mathrm{B}_{\mathrm{s}}^{0} \rightarrow \mathrm{J} / \psi \phi$ channel with first LHCb data
}

\section{Basem Khanji*}

CPPM, Aix Marseille Université,CNRS/in2p3, Maseille, France

E-mail: khanji@cppm.in2p3.fr

\begin{abstract}
We discuss the measurement of the phase $\phi_{\mathrm{s}}$ in the $\mathrm{B}_{\mathrm{s}}^{0} \rightarrow \mathrm{J} / \psi \phi$ decay channel at the LHCb experiment. The Standard Model expectation for this parameter is very small, whereas models of New Physis predict higher values. We describe the strategy developed on Monte Carlo to perform this measurement and present the first studies carried out on data taken in 2010 at $7 \mathrm{TeV}\left(\sim 36 \mathrm{pb}^{-1}\right)$.
\end{abstract}

Workshop on Discovery Physics at the LHC-Kruger 2010

December 05-10, 2010

Kruger National Park, Mpumalanga, South Africa

\footnotetext{
* Speaker.
} 


\section{Theoretical introduction}

Neutral mesons transform into their anti-particles via the weak interaction, this process is called flavor oscillation. The decay $\mathrm{B}_{\mathrm{s}}^{0} \rightarrow \mathrm{J} / \psi \phi$ involves two main phases; $\Phi_{\mathrm{M}}$ the mixing phase and $\Phi_{\mathrm{D}}$ the decay phase. The interference between decays, either directly or via oscillation, gives rise to $\phi_{\mathrm{S}}\left(=\Phi_{\mathrm{M}}-2 \Phi_{\mathrm{D}}\right)$. This phase is related to the smallest angle in the b-s unitarity triangle $\left(\beta_{\mathrm{s}}\right) ; \phi_{\mathrm{s}} \sim-2 \beta_{\mathrm{s}}$. This is particularly important, as this angle $\beta_{\mathrm{s}}$ is predicated to be small within the Standard Model framework; $\beta_{\mathrm{s}}=(0.036 \pm 0.0017) \mathrm{rad}[1]$. Any deviation, in this measurement, from the Standard Model prediction will be a clear sign of New Physics effects in this channel.

Experimentally, this phase is accessible via the differential decay rates for the $\mathrm{B}_{\mathrm{s}}^{0} \rightarrow \mathrm{J} / \psi \phi$ channel, where three angular variables are needed to disentangle the final state superposition [2]. This superposition is due to the orbital spin conservation between the initial $\mathrm{B}_{\mathrm{s}}^{0}$ (pseudo-scalar particle) state and the final state $\mathrm{J} / \psi \phi$ (vector particles), Figure 1 shows the transversity basis in which the angular variables are defined.

In Section 2, we give a concise description of the LHCb detector. In Section 3 we present the $\phi_{\mathrm{S}}$ analysis strategy at LHCb. In Section 4, we present the expected sensitivity based on Monte Carlo studies. Finally, in Section 5 we give the state of art for the analysis on 2010 real data $^{1}$.
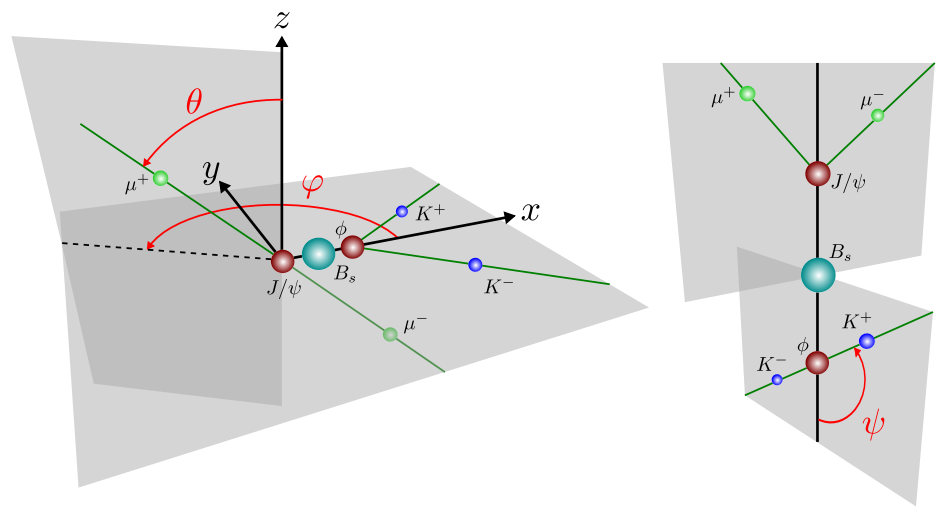

Figure 1: Representation of the angles in the transversity basis for the $\mathrm{B}_{\mathrm{s}}^{0} \rightarrow \mathrm{J} / \psi \phi$ channel. Left : $\mathrm{J} / \psi$ rest frame. Right : $\phi$ rest frame.

\section{2. $\mathrm{LHCb}$ detector}

$\mathrm{LHCb}$ is one of the four large LHC experiments. It is dedicated to searching for new physics phenomena in the domain of CP violation and rare B decays. LHCb detector is shown in Figure 1.

\footnotetext{
${ }^{1}$ During the Kruger 2010 conference, the work to analyse 2010 data was still in progress.
} 
The $\mathrm{LHCb}$ is a forward spectrometer, covering the region from 10 to $300 \mathrm{mrad}$ relative to beam axis. $\mathrm{LHCb}$ detectors contain the following main elements :

- VELO or vertex locator : surroundings the interaction region. It is made up of disks of silicon, perpendicular to the beam axis. The VELO reconstructs the position of primary and secondary vertexes with an excellent precision;

- the tracking system : based around large dipole magnet, with a trigger tracker station and three tracking stations;

- two RICH detectors : identifies the particles with different momentum ranges, separating pions from kaons. This is a very useful for tagging the initial flavor of the B mesons. Figure 2 (right) shows the very good kaon-pion separation achieved in data;

- calorimeter system: identifies electrons, hadrons and neutrals;

- muon detector: uses five stations of detectors for detecting muons.

In 2010, the LHC machine's configuration was set to maximize the number of visible interaction per bunch. This had the benefit of deliviering more luminosity than in the default configuration. At the same time this increases the number of tracks and vertexes per event, creating a challenging environment for the trigger and reconstruction systems. Indeed the rate of "pile-up" events sometimes reached five times the LHCb design value.
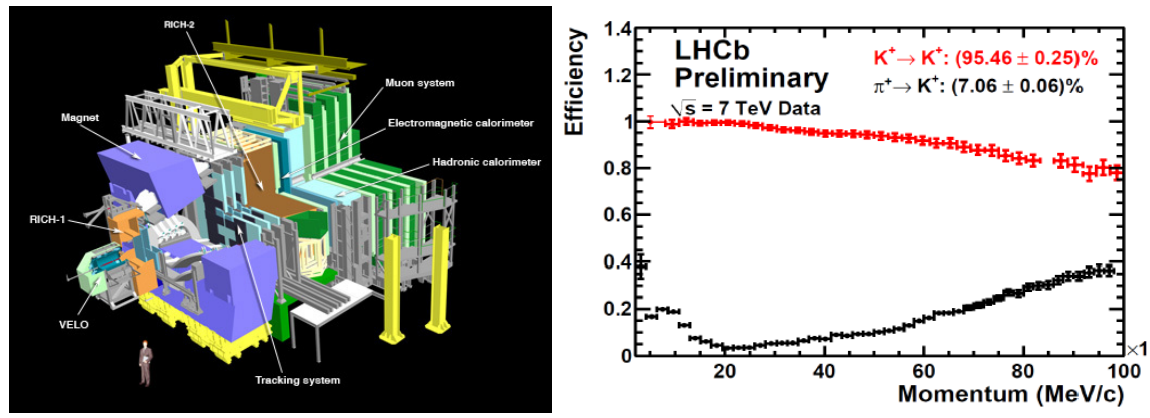

Figure 2: Left : layout of the $\mathrm{LHCb}$ spectrometer. Right : performance with real data for kaon-pion misidentification rates.

\section{3. $\phi_{\mathrm{s}}$ measurement analysis strategy}

The analysis strategy [3] developed in LHCb for the first data analysis is as follows :

- trigger and select the $\mathrm{B}_{\mathrm{s}}^{0} \rightarrow \mathrm{J} / \psi \phi$ events;

- measure the proper time of the events;

- measure the tranversity angles of the decay products of the events;

- tag the initial flavor of the selected $\mathrm{B}_{\mathrm{s}}^{0}$ in the event; 
- fit the phase $\phi_{\mathrm{s}}$.

This strategy implies the need for excellent proper time resolution to resolve the fast $\mathrm{B}_{\mathrm{s}}^{0}$ oscillations. In addition, a good flavor tagging efficiency with a low and well known mistag rate is required. Finally, it is necessary to control the angular distortions ${ }^{2}$ to reduce the systematics induced.

\section{4. $\phi_{\mathrm{S}}$ measurement studies with Monte Carlo}

\subsection{Trigger and selections}

We summarize, in this section, the main points of the selection strategy, detailed in [4]. We intend to use the trigger lines which are fired mainly by muons objects and do not bias the proper time of the $\mathrm{B}_{\mathrm{s}}^{0}$. The total trigger efficiency is $\sim 70 \%$, measured using Monte Carlo. An approach for using the biased lines is being investigated. The main purposes of the selection are :

- maximizing the signal annual yield and reducing the background level;

- minimizing biases on proper time and angular distributions, hence reducing the systematics on the fitted physical parameters;

- selecting the $\mathrm{B}$ mesons in $\mathrm{B}_{\mathrm{s}}^{0} \rightarrow \mathrm{J} / \psi \phi$ and its control channels $\mathrm{B}^{0} \rightarrow \mathrm{J} / \psi \mathrm{K}^{* 0}$ and $\mathrm{B}^{+} \rightarrow \mathrm{J} / \psi \mathrm{K}^{+}$ in the same way. In doing so, we ensure the selected events share the same phase space, and same tagging properties.

The selection places emphasis on kinematic variables (e.g. $p_{\mathrm{T}}$ and mass cuts), variables based on reconstruction quality (e.g. $\chi^{2}$ of tracks and vertexes) and avoids any cuts on variables correlated with the proper time distributions.

The $\mathrm{J} / \psi$ selection suppresses a huge combinatoric background. Events surviving the selection requirements are used as input for the selection of $\phi$ particle, finally the $\mathrm{B}_{\mathrm{s}}^{0}$ mesons are reconstructed using the accepted $\mathrm{J} / \psi$ and $\phi$.

The main background source in this channel are prompt events where the $\mathrm{J} / \psi$ comes from primary vertex. This type of background has, however, a negligible effects on the global fit. The other main source of the background, composed of long-lived particles, has an impact on the sensitivity of the fitted parameters. Using Monte Carlo at $7 \mathrm{TeV}$, we expect $\sim 30000$ events $^{3}$ per $1 \mathrm{fb}^{-1}$. The distortion on the angular distributions is below $8 \%$, which is caused mainly by the geometrical acceptance of the detector.

\subsection{Tagging}

Tagging the initial flavor of the $\mathrm{B}_{\mathrm{s}}^{0}$ at production is indispensable piece of this analysis. Although the untagged decay rates do carry information on $\phi_{\mathrm{s}}$, the sensitivity of the tagged rates is much higher, particularly in the case $\phi_{\mathrm{s}}$ is small.

The identification of the initial flavor of the reconstructed $\mathrm{B}$ mesons is performed at $\mathrm{LHCb}$ by different flavor tagging algorithms, described in [5]. As stated in these references, the tagging

\footnotetext{
${ }^{2}$ deviations from theoretical angular projections due to experimental effects like reconstruction, detector shape.

${ }^{3}$ In [3] results were obtained for $\sqrt{s}=14 \mathrm{TeV}$ and $2 \mathrm{fb}^{-1}$ and have been scaled here to $\sqrt{s}=7 \mathrm{TeV}$ and $1 \mathrm{fb}^{-1}$.
} 
algorithm is characterized by the misatg rate $(\omega=W /(W+R))$, tagging efficiency $\left(\varepsilon_{\text {tag }}=(W+\right.$ $R /(W+R+U)))$ and the effective tagging efficiency $\left(\varepsilon_{\text {eff }}=\varepsilon_{\text {tag }}(1-2 \omega)^{2}\right)$; where $\mathrm{W}, \mathrm{R}$ and $\mathrm{U}$ are, respectively, the number of events wrongly tagged, correctly tagged and untagged. The combined effective tagging efficiency measured on Monte Carlo is $5.3 \%$ for the $\mathrm{B}_{\mathrm{s}}^{0} \rightarrow \mathrm{J} / \psi \phi$ channel. Tuning and calibration of the tagging algorithm will be executed in the $\mathrm{B}^{0} \rightarrow \mathrm{D}^{*-} \mu^{+} v, \mathrm{~B}^{+} \rightarrow \mathrm{J} / \psi \mathrm{K}^{+}$, $\mathrm{B}^{0} \rightarrow \mathrm{J} / \psi \mathrm{K}^{* 0}$ and $\mathrm{B}_{\mathrm{s}}^{0} \rightarrow \mathrm{D}_{\mathrm{s}}^{-} \pi^{+}$channels.

\subsection{Fit}

This procedure is explained extensively here [6]. The differential decay rates for the $\mathrm{B}_{\mathrm{s}}^{0} \rightarrow$ $\mathrm{J} / \psi \phi$ channel is a function with 8 physical parameters; the weak phase $\phi_{\mathrm{s}}$, the mass and width difference between the $\mathrm{B}_{\mathrm{s}}^{0}$ physical and flavor states $\Delta \Gamma_{\mathrm{s}}$ and $\Delta m_{\mathrm{s}}$, two polarization amplitudes of the final state, $\left|A_{\perp}(0)\right|^{2},\left|A_{\|}(0)\right|^{2}$, in addition to their strong phases $\delta_{\perp}$ and $\delta_{\|}$. It also contains 6 observables; mass, proper time, three angular variables and the $\mathrm{B}_{\mathrm{s}}^{0}$ flavor tagging decision.

Background contributions from both prompt and long lived components will be described using the side bands of the $\mathrm{B}_{\mathrm{s}}^{0}$ invariant mass distribution. Angular variable distortions are calculated from Monte Carlo and cross checked from the $\mathrm{B}^{0} \rightarrow \mathrm{J} / \psi \mathrm{K}^{* 0}$ channel. The proper time resolution model is estimated with the help of prompt background. Wrong tag fractions are also taken into account.

Using Monte Carlo pseudo experiments, the statistical error on $\phi_{\mathrm{s}}$ is estimated to be $0.07 \mathrm{rad}$ with $1 \mathrm{fb}^{-1}$ of data taken at $\sqrt{s}=7 \mathrm{TeV}$. Expected sources of systematics errors are the proper time resolution modeling, the angular distortions and the mistag fraction, as shown in Table 1. Those errors are obtained from extensive Monte Carlo and Monte Carlo pseudo experiments studies. No limiting systematics to this measurement are anticipated.

\begin{tabular}{|l|c|c|}
\hline Parameter & Variation & $\left|\phi_{\mathrm{s}}^{\text {wrong }}-\phi_{\mathrm{s}}^{\text {true }}\right| / \phi_{\mathrm{s}}^{\text {true }}$ \\
\hline \hline Angular distortions & $\pm 5 \%$ & $7 \%$ \\
Proper time resolution & $\pm 5 \mathrm{fs}$ & $6 \%$ \\
Mistag & $1 \%$ & $7 \%$ \\
\hline
\end{tabular}

Table 1: Relative systematic variation on $\phi_{\mathrm{s}}$ (column 3), due to a parameter variations (columns 2) (from [3])

\section{5. $\phi_{\mathrm{s}}$ with 2010 real data}

LHCb has collected $\sim 37 \mathrm{pb}^{-1}$ of data in 2010 at $7 \mathrm{TeV}$. The $\mathrm{B}_{\mathrm{s}}^{0} \rightarrow \mathrm{J} / \psi \phi$ yield is $\sim 900$ events in $33 \mathrm{pb}^{-1}$. Figure 4 shows the mass distribution for this channel, where the cut $t>0.3 \mathrm{ps}$ was applied to remove the huge prompt background illustrating the $\mathrm{B}_{\mathrm{s}}^{0}$ mass peack.

The effective tagging efficiency calculated in $\mathrm{B}^{0} \rightarrow \mathrm{D}^{*-} \mu^{+} v$ channel is at present $\sim 2 \%$, which is $60 \%$ of the expected performance obtained in Monte Carlo for this channel. Figure 3 (left) shows the asymmetry plot for this channel using $\sim 3 \mathrm{pb}^{-1}$ real data sample. As the $\mathrm{B}^{+}$meson does not oscillate, the mistag rate, in $\mathrm{B}^{+} \rightarrow \mathrm{J} / \psi \mathrm{K}^{+}$control channel, can be easily obtained by comparing the tagging decision given by the taggers, with the flavor of the signal $\mathrm{B}$ at decay using the $\mathrm{K}$ charge. With $36 \mathrm{pb}^{-1}$, the 2010 real data sample contains $\sim 10000$ events, as seen in Figure 4 (centre). For 


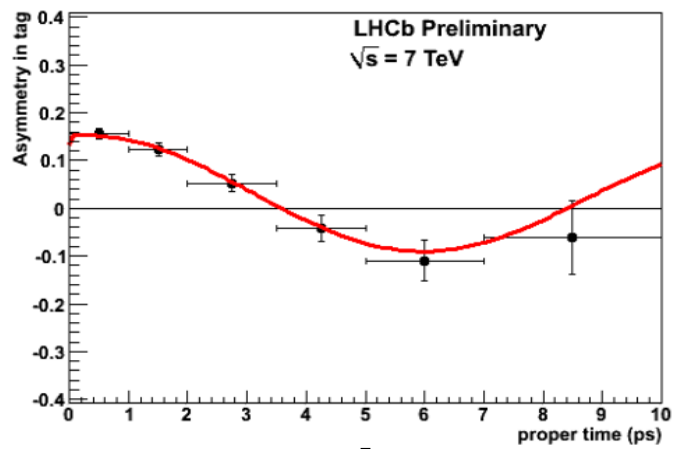

Figure 3: $\mathrm{B}^{0} \rightarrow \mathrm{D}^{*-} \mu^{+} v$ oscillation plot using $\sim 3 \mathrm{pb}^{-1}$ of 2010 data.

the $\mathrm{B}^{0} \rightarrow \mathrm{J} / \psi \mathrm{K}^{* 0}$ channel, one obtains, using the same integrated luminosity, $\sim 4000$ events, as seen in Figure 4 (left). The mistag fraction has to be calculated from a fit to the time-dependent asymmetry.

The proper time resolution as measured with the VELO, is $\sim 50 \mathrm{fs}$, which is more than adequate for resolving the very fast $\mathrm{B}_{\mathrm{s}}^{0}$ oscillations.

Since the Kruger 2010 conference, much progress in this analysis has been achieved and new results have been reported $[7,8,9,10]$. Further upates are expected soon.
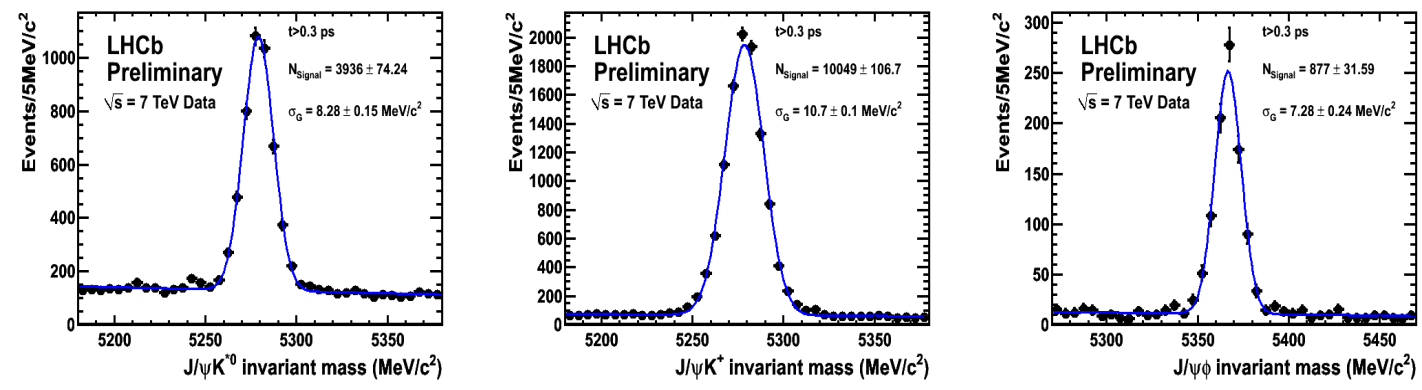

Figure 4: Left : the control channel $\mathrm{B}^{0} \rightarrow \mathrm{J} / \psi \mathrm{K}^{* 0}$ mass distribution. Centre : the control channel $\mathrm{B}^{+} \rightarrow$ $\mathrm{J} / \psi \mathrm{K}^{+}$mass distribution. Right $: \mathrm{B}_{\mathrm{s}}^{0} \rightarrow \mathrm{J} / \psi \phi$ mass distribution using real data sample.

\section{Conclusion}

The $\phi_{\mathrm{S}}$ measurement is one of the key analysis that LHCb will perform. The Standard Model expectation for this parameter will be tested and New Physics will be probed. The decay channel $\mathrm{B}_{\mathrm{s}}^{0} \rightarrow \mathrm{J} / \psi \phi$ is the golden channel for $\phi_{\mathrm{s}}$ measurement, its analysis strategy has been developed using the LHCb Monte Carlo simulation and is being applied to real data. $\sim 900 \mathrm{~B}_{\mathrm{s}}^{0} \rightarrow \mathrm{J} / \psi \phi$ events have been collected with an integrated luminosity of $36 \mathrm{pb}^{-1}$. Work is under way to make the first determination of $\phi_{\mathrm{s}}$ using 2010 data. The much larger samples anticipated in 2011-2012 will allow for very high precision. 


\section{References}

[1] CKMfitter groupe, J. Charles et al., Eur. Phys. J. C41, 1-131 (2005).

[2] A. Dighe et al., "Angular distribution and lifetime difference in $\mathrm{B}_{\mathrm{s}}^{0} \rightarrow \mathrm{J} / \psi \phi$ decays", Phys. Lett. B 369, 144 (1996).

[3] Adeva, B et al., "Roadmap for selected key measurements of LHCb", LHCb-PUB-2009-029, [arXiv:0912.4179v3], Dec (2009).

[4] Calvi, $M$ et al., "Lifetime Unbiased Selection of $B_{s} \rightarrow J / \psi \phi$ and related control channels: $B_{d} \rightarrow J / \psi K^{*}$ and $B^{+} \rightarrow J / \psi K^{+}$, LHCb-2009-025. CERN-LHCb-2009-025 (2009).

[5] M. Calvi et al., "Flavour Tagging Algorithms and Performances in LHCb", LHCb note 2007-058, (2007).

[6] C. Langenbruch et al., "Fit of the decay $\mathrm{B}_{\mathrm{s}}^{0} \rightarrow \mathrm{J} / \psi \phi$ ", LHCb-PUB-2009-028 (2009).

[7] The LHCb Collaboration, "b-hadron lifetimes measurement with exclusive $b \rightarrow J / \psi X$ decays reconstructed in the 2010 data", LHCb-CONF-001-2011, (2011).

[8] The LHCb Collaboration," Untagged angular analysis of $B_{d} \rightarrow J / \psi K^{*}$ and $\mathrm{B}_{\mathrm{s}}^{0} \rightarrow \mathrm{J} / \psi \phi$ decays ", LHCb-CONF-002-2011, (2011).

[9] The LHCb Collaboration,'Optimization and caliberation of the LHCb tagging performance using 2010 data", LHCb-CONF-003-2011, (2011).

[10] The LHCb Collaboration,"Measuremnt of $\Delta M_{s}$ in the decay $B_{s}^{-} \rightarrow D_{s}^{-}\left(K^{+} K^{-} \pi^{-}\right)(3) \pi^{\prime \prime}$, LHCb-CONF-005-2011, (2011). 